in the average rate of loss of heat by convection, and consequently there is a fall in the temperature, and hence in the resistance, of the grid. The fall in resistance can be measured by a Wheatstone bridge method. The way in which it is suggested that this process may be utilised in the construction of a standard source of sound is as follows. The source is made in the form of a cylindrical Helmholtz resonator-say from brass tubing about 2 in. diameter. At one end is fitted a telephone in such a way that the diaphragm forms part of the inner wall of the resonator, while at the other end is the orifice carrying the hot-wire grid. If the telephone diaphragm is made to vibrate by current from a thermionic valve oscillator, and the frequency of the current is adjusted until it is equal or nearly equal to that of the resonator, a pure tone of moderate intensity issues from the orifice. At the same time the hot-wire grid suffers a change of resistance which provides a measure of the amplitude of the vibration in the orifice, and hence of the strength of the source. With a standard pattern grid and holder the change in resistance is about I $2 \mathrm{ohms}$ when a sound of pitch 200 vibrations/sec. is produced, which is loud enough to be just audible at a distance of ro feet-the source being placed on the ground in the open. The strength of the source can be varied at will by using a variable series resistance or a shunt with the telephone. The use of resonance serves to purify the note which comes from the telephone, but it is desirable in addition that the oscillator should be of such a type that the telephone note is already fairly pure. The use of a very impure note may lead to poor results, as the resistance change will then depend only partly on the amplitude of the fundamental.

It may be noted that a simple method is known of obtaining the relation between the change in resistance of a grid and the amplitude and frequency of the vibration producing it-the motion being simple harmonic. Hence, if the frequency of the vibration is known, the strength of the source (defined as the rate at which fluid is introduced or abstracted at the source) can be found in absolute measure. The amplitude of the waves-or, if preferred, the flux of energy - in the surrounding medium can then be calculated in certain cases. A simple case is when the source is close to a rigid plane.

Any other means of producing a suitable sound can be used in place of the telephone diaphragm provided it is small enough to go inside a resonator. If the damping factor of the resonator has been determined experimentally, the acoustical output of the primary source inside the resonator can be calculated from the indications of the hot-wire grid in the orifice. The output of the internal source includes. ( $\mathrm{I}$ ) the radiation of acoustical energy from the orifice, which has been dealt with above, and (2) work done against viscous forces in the orifice. Unless the orifice is large, (2) is far the more important part, and the radiation losses may be negligible by comparison.

Signals Experimental Establishment, E. T. PARIS. Woolwich, S.E.18, Sept. 2.

\section{Occurrence of the Rare Whale, Mesoplodon Layardi, on the Tasmanian Coast.}

Some years ago, on July 30, I9I8, my friend Mr. G. H. Smith of Leprena, Southern Tasmania, brought me word that a beaked whale, "without teeth," had been cast up on the beach at Recherche Bay near his property, that he had already removed the blubber, and that the carcase still remained on the shore. Being occupied at the time with university teaching I was unable to visit. Recherche Bay till some weeks later. High seas were then running, and the remains had been lifted by these and thrown farther up on the basaltic boulders which strew the beach at this spot. This resulted in some considerable damage to the skeleton. Nearly all the ribs were broken, some into three or four pieces, and many of the neural spines had been smashed from the vertebrae. The skull . was also damaged, although some of the flesh and integument was still adhering.

The body was naturally in a somewhat decayed and pulpy condition, but with Mr. Smith's help I was able to salve the remains and so obtain the skeleton. This is almost complete and is now in my Department.

My friend was good enough to hand me some measurements and notes which he had made and these I reproduce. The animal "was a female whale. The total length was about I $8 \mathrm{ft}$; ; its jaws were $2 \mathrm{ft}$. 6 in. long, about 4 in. in diameter at the end, no teeth above the gums. Fins $2 \mathrm{ft}$. long, 8 in. wide, and tapered, not round like the black whale type. Its flukes were about $4 \mathrm{ft}$. wide; it had the appearance of a fast fish as it was rather thin in the body. There was a small fin on its hump, about a foot high, with a decided rake towards the tail. The colour the same as the sperm whale, dark grey and light underneath." Mr. Smith further states that the blubber yielded 50 gallons of oil of the finest quality, and that he believes that the animal was driven ashore by "killers," of which there were a number in the bay at the time. There were, however, no marks of injury on the body.

The matter of recording this specimen seems called for, particularly in view of the description by $\mathrm{Mr}$. E. R. Waite, director of the South Australian Museum (Rec. S. Aus. Mus., vol. ii., No. 2), of the discovery, on the South Australian coast, of an immature male of this species of Mesoplodon. The Tasmanian specimen was a female and mature, as is witnessed by the condition of the skeleton and by the fact that the pulp cavity of the tooth is entirely closed below.

The form of the tooth corresponds exactly with that figured by Gray for his Callidon güntheri (Ann. Mag. Nat. Hist., I87I), which, as Flower and Turner suggested, was a female of the present species. It seems now that we must conclude from Waite's description that the mature condition of the tooth in the female represents a stage which is early passed through in the male.

No pelvic bones were discovered, nor was there any trace of the denticles found in the integument of the jaws of other species. The oil has a density of 0.88 at $12.5^{\circ} \mathrm{C}$. This whale has now been recorded from the coast of every Australian State except Victoria and West Australia.

T. THOMSON
University of Tasmania, Hobart, July 20.

\section{Atoms and Electrons.}

ON the basis of any theory of atomic structure which classifies the elements according to rare gas type, cerium and thorium should be comparable with one another, since the atoms of each are possessed of four electrons more than those of the corresponding inert gases, xenon and niton respectively. There are, however, in the thorium atom, thirty-two more extra-nuclear electrons than in the cerium atom. In spite of this fact, it appears that the distances between atomic centres in crystals of these elements are practically the same $(\mathrm{Ce}=3.62 \mathrm{~A} . \mathrm{U}$. and $\mathrm{Th}=3.56 \AA$ A.U., according to Hull), the distance being, if anything, slightly the 